OPEN ACCESS

Edited by:

Ken J. Ishii,

University of Tokyo, Japan

Reviewed by:

Eda K. Holl,

Duke University, United States

Eun Jeong Park,

Mie University, Japan

*Correspondence:

Francis O. Eko

feko@msm.edu

tThese authors have contributed equally to this work

Specialty section: This article was submitted to Microbial Immunology,

a section of the journal

Frontiers in Immunology

Received: 02 November 2020 Accepted: 29 January 2021

Published: 22 February 2021

Citation:

Howard S, Richardson S, Benyeogor I, Omosun Y, Dye K, Medhavi F, Lundy S, Adebayo O, Igietseme JU and Eko FO (2021) Differential miRNA Profiles Correlate With Disparate Immunity Outcomes Associated With Vaccine

Immunization and Chlamydial

Infection. Front. Immunol. 12:625318. doi: 10.3389/fimmu.2021.625318

\section{Differential miRNA Profiles Correlate With Disparate Immunity Outcomes Associated With Vaccine Immunization and Chlamydial Infection}

\author{
Simone Howard ${ }^{1 \dagger}$, Shakyra Richardson ${ }^{1 \dagger}$, Ifeyinwa Benyeogor ${ }^{2}$, Yusuf Omosun ${ }^{1}$, \\ Kamran Dye ${ }^{3}$, Fnu Medhavi ${ }^{1}$, Stephanie Lundy ${ }^{1}$, Olayinka Adebayo ${ }^{1}$, \\ Joseph U. Igietseme ${ }^{4}$ and Francis O. Eko ${ }^{\text {1* }}$ \\ ${ }^{1}$ Department of Microbiology, Biochemistry and Immunology, Morehouse School of Medicine, Atlanta, GA, United States, \\ ${ }^{2}$ Winship Cancer Institute, Emory University School of Medicine, Atlanta, GA, United States, ${ }^{3}$ Department of Chemistry, \\ Morehouse College, Atlanta, GA, United States, ${ }^{4}$ Centers for Disease Control and Prevention (CDC), Atlanta, GA, \\ United States
}

Vaccine-induced immune responses following immunization with promising Chlamydia vaccines protected experimental animals from Chlamydia-induced upper genital tract pathologies and infertility. In contrast, primary genital infection with live Chlamydia does not protect against these pathologies. We hypothesized that differential miRNA profiles induced in the upper genital tracts (UGT) of mice correlate with the disparate immunity vs. pathologic outcomes associated with vaccine immunization and chlamydial infection. Thus, miRNA expression profiles in the UGT of mice after Chlamydia infection (Live EB) and immunization with dendritic cell (DC)-based vaccine (DC vaccine) or VCG-based vaccine (VCG vaccine) were compared using the NanoString nCounter Mouse miRNA assay. Of the 602 miRNAs differentially expressed (DE) in the UGT of immunized and infected mice, we selected 58 with counts $>100$ and $p$-values $<0.05$ for further analysis. Interestingly, vaccine immunization and Chlamydia infection induced the expression of distinct miRNA profiles with a higher proportion in vaccine-immunized compared to Chlamydia infected mice; DC vaccine (41), VCG vaccine (23), and Live EB (15). Hierarchical clustering analysis showed notable differences in the uniquely DE miRNAs for each experimental group, with DC vaccine showing the highest number (21 up-regulated, five down-regulated), VCG vaccine (two up-regulated, five down-regulated), and live EB (two up-regulated, four down-regulated). The DC vaccine-immunized group showed the highest number (21 up-regulated and five down-regulated compared to two upregulated and four down-regulated in the live Chlamydia infected group). Pathway analysis showed that the DE miRNAs target genes that regulate several biological processes and functions associated with immune response and inflammation. These results suggest that the induction of differential miRNA expression plays a significant role in the disparate immunity outcomes associated with Chlamydia infection and vaccination.

Keywords: Chlamydia, immunization, infection, microRNA, immunity, pathology 


\section{INTRODUCTION}

Chlamydia trachomatis genital infection is the commonest bacterial cause of sexually transmitted disease (STD) worldwide. Although both men and women are affected, the infection is usually more severe in women. If untreated, it can lead to irreversible complications characterized by pelvic inflammatory disease (PID), ectopic pregnancy, and tubal factor infertility (1-3). About $33 \%$ of C. trachomatis infection-associated PID occurs mainly in reproductive-age women and on examination, presents as pelvic or lower abdominal pain or uterine or adnexal tenderness $(4,5)$. Acute PID resulting from genital Chlamydia infection is often associated with more extended hospitalization stays, increased inflammatory markers, higher incidence of tubo-ovarian abscess, ectopic pregnancy, and tubal factor infertility (6). Tubal infertility results from tubal damage caused by inflammatory fibrosis (scarring) that lead to tubal occlusion (7). Recent studies show that Chlamydia-induced fibrosis and fertility-related epithelial dysfunction is associated with the pathologic process of epithelial-mesenchyme transition (EMT) (8).

There is currently no licensed human Chlamydia vaccine despite concerted efforts. We have developed several Chlamydia vaccine candidates, including a dendritic cell (DC)-based experimental cellular vaccine comprising IL-10 knockout (KO) mouse bone-marrow derived DC cultured for $12 \mathrm{~h}$ with UVirradiated Chlamydia EBs (DC vaccine) and a Vibrio cholerae ghost (VCG)-based subunit vaccine consisting of the chlamydial polymorphic membrane protein $\mathrm{D}(\mathrm{PmpD})$ and porin $\mathrm{B}$ (PorB) proteins packaged in genetically derived $V$. cholerae cell envelops (VCG vaccine). We previously showed that immunization with DC or VCG vaccine protected mice from Chlamydiainduced upper genital tract (UGT) pathologies and infertility. In contrast, immunization with live Chlamydia elementary bodies (EBs) failed to protect against these pathologies following a subsequent genital challenge infection $(9,10)$. This observation is quite intriguing since immunization with these experimental vaccines and primary genital infection with live Chlamydia both induce a significant degree of protective immunity in the lower genital tract marked by lower microbial burden and shortened infection (9-11). Nevertheless, these studies revealed that functionally, vaccine-induced immune responses differ from Chlamydia infection-induced immune responses since they lead to different outcomes in the UGT. The efficacy of the DC vaccine is a function of the propensity of their antigen-presenting cells (APCs) to rapidly and preferentially activate a high magnitude of IFN- $\gamma$-secreting $\mathrm{T}$ cells and is associated with the induction of a persistent high Th1 cell frequency in the genital tract (11). Distinct features of Chlamydia-induced UGT pathology in mice are hydrosalpinx development and tubal dilatation, leading to tubal occlusion and infertility (10). We and others have shown that many of these pathological lesions occur at later time points in the absence of cultivable bacteria $(10,12)$. Although C. trachomatis infection stimulates a CD4 $+\mathrm{T}$ cell response to protect against reinfection (13), T cell immunity appears to be short-lived in humans. It may also influence the development of immunopathology (14).
Several mechanisms have been proposed for the inflammatory response and associated consequences in Chlamydia infected host cells, including the presence of chlamydial proteins and host-derived small non-coding RNAs (miRNAs) expressed during C. trachomatis infection (8, 15-18). MicroRNAs have emerged as important regulators of biological pathways, including immune response and inflammation by modifying gene regulatory networks (19). miRNAs are 18-22 nucleotides long, endogenous, non-coding RNAs whose primary function is RNA silencing and post-transcriptional regulation of gene expression, which is accomplished by either repressing translation or inducing mRNA degradation, depending on its sequence homology with the targeted mRNA $(19,20)$. Accordingly, complete complementarity between the miRNA and its target mRNA leads to cleavage and degradation, while mismatches lead to suppression of translation. Predictions by computational analysis suggest that each miRNA molecule can target 100 or more transcripts and that multiple miRNA species may regulate a single mRNA (21).

Moreover, functional studies indicate that miRNAs regulate over $30 \%$ of mammalian gene expression, involving essentially every cellular process so far investigated. These processes include cellular differentiation, maintenance of cellular integrity, development, functions and normal metabolism, reproduction, and several pathologic processes and diseases, such as fibrosis and oncogenesis (22). MiRNAs also regulate mitochondrial function, which is necessary for C. trachomatis development (23). The differential expression of miRNAs during ocular and genital chlamydial infection has also been severally reported $(15,16,23-$ 26). Specific miRNA expression can predict the development of Chlamydia infection-induced PID (27). The relative degree of virulence of the infecting Chlamydia is associated with the host miRNA expression profile, determining disease severity (18). Besides, immune cell type-specific miRNAs appear to regulate the immune response to chlamydial infection $(16,26)$. Thus, miRNAs play a significant role in both the quality of the host immune response and the development of complications following Chlamydia infection. In this study, we examined if infection with live Chlamydia or immunization with a VCG or DC chlamydial vaccine drives differing miRNA levels and diversity in the upper genital tract of mice. Thus, we performed a comparative assessment of the host miRNAs differentially expressed in the upper genital tract (UGT) tissues of mice after infection with live Chlamydia and immunization with Chlamydia vaccines using the NanoString nCounter Mouse miRNA assay. We showed that vaccine immunization and live Chlamydia infection induced a diversity of uniquely differentially expressed miRNAs in the UGT tissues of mice. In addition, we identified candidate miRNAs whose up or downregulation may account for the observed differences between live Chlamydia infection- and chlamydial vaccine-induced immunity.

\section{MATERIALS AND METHODS}

\section{Ethics Statement}

In this study, the recommendations contained in the Guide for the Care and Use of Laboratory Animals of the National 
Institutes of Health were followed. The Institutional Animal Care and Use Committee (IACUC) of Morehouse School of Medicine (MSM) (Assurance number A3381-01) approved the study protocol (Protocol Number: 16-15). MSM-IACUC adheres to the National Institute of Health (NIH) guidelines for the care and use of laboratory animals, the Public Health Service (PHS) policy, and the Animal Welfare Act.

\section{Animals}

Six to 7-week-old female C57BL/6J mice (stock number 000664) obtained from The Jackson Laboratory (Bar Harbor, ME) were used in this study and were allowed to acclimate for 10 days in the MSM animal facility prior to experimentation. The animals were fed food and provided water ad-libitum and maintained in laminar flow racks under pathogen-free conditions with a $12 \mathrm{~h}$ light and $12 \mathrm{~h}$ dark cycle. All infections, immunizations and surgery were performed under isoflurane anesthesia, and all efforts were made to minimize suffering. Mice, placed in a chamber were exposed to $2-4 \%$ isoflurane in a fume hood for $30 \mathrm{~min}$. Mice were euthanized by carbon dioxide asphyxiation, followed by cervical dislocation as recommended by the Panel on Euthanasia of the American Veterinary Medical Association.

\section{Chlamydia Stocks and Antigens}

In stock preparations of Chlamydia muridarum Nigg (the agent of mouse pneumonitis) used in this study were propagated on HeLa cell monolayers followed by purification of elementary bodies (EBs) over renografin gradients and stored at $-70^{\circ} \mathrm{C}$ (Division of Scientific Research, Centers for Disease Control and Prevention, Atlanta), according to standard procedures. Chlamydia stock titers were expressed as inclusion-forming units (IFU) per milliliter (9). UV-inactivated Chlamydia was prepared by exposing aliquots of purified EBs in Eppendorf tubes to UV radiation for $1-2 \mathrm{~h}$ and stored at $-70^{\circ} \mathrm{C}$ until used.

\section{Production of rVCG and IL-10KO DC Vaccines}

$V$. cholerae $\mathrm{O} 1$ strain $\mathrm{H} 1$ harboring the lysis plasmid pDKLO1 was transformed with plasmids expressing $\mathrm{pPmpD}$ or pMALPorB and cultured in brain heart infusion (BHI) broth at $37^{\circ} \mathrm{C}$. The rVCG vaccines were obtained by genetic inactivation of the $V$. cholerae cells as previously described $(28,29)$. The process involves the generation of a lysis tunnel structure through the bacterial cell envelope complex by lysis protein E, which leads to the cellular release of cytoplasm, producing cell envelopes devoid of cellular content. The culture was centrifuged to harvest the cell envelopes with the packaged $\mathrm{PmpD}$ and PorB proteins (VCG vaccine), which were washed, freeze-dried and stored refrigerated until used. IL-10KO DC $\left(2 \times 10^{5}\right)$ were isolated from IL-10KO mice by standard protocols and pulsed for $12 \mathrm{~h}$ with UV-inactivated C. muridarum EBs $\left(1 \times 10^{5} \mathrm{IFU}\right)$ as described previously (11). This constituted the IL-10KO DC-based cellular vaccine (DC vaccine).

\section{Vaccine Immunization and Chlamydia Infection of Mice}

Naive female C57BL/6 wild-type mice (4/group) were immunized intranasally (IN) with $500 \mu \mathrm{g}$ each of lyophilized rVCG-PorB and rVCG-PmpD ( $1 \mathrm{mg}$ total) in $25 \mu \mathrm{l}$ PBS twice 2 weeks apart. Another group (4/group) was adoptively immunized intravenously via the tail vein with UV-irradiated Chlamydia EB pulsed for $12 \mathrm{~h}$ with IL-10KO DC $\left(2.5 \times 10^{7}\right.$ cells/mouse $)$ (11) in $200 \mathrm{ml}$ of PBS (DC vaccine). A third group of mice was infected by intravaginal administration of $1 \times 10^{5}$ IFU of live C. muridarum EBs, 5 days after subcutaneous injection with $2.5 \mathrm{mg}$ Depo-Provera (medroxyprogesterone acetate; Pharmacia \& Upjohn Co., NY). A group immunized intravaginally with $50 \mu \mathrm{l}$ of PBS alone served as control. Each group contained three mice and all immunizations were administered while under isoflurane (2-4\%) anesthesia. Mice were euthanized 2 weeks after immunization or infection and the entire genital tract was harvested, and the cervix (lower genital tract) and uterine horns (upper genital tract) were separated and frozen immediately on dry ice and stored at $-80^{\circ} \mathrm{C}$.

\section{RNA Isolation}

Harvested genital tract tissues were each homogenized using the gentleMACS Dissociator in combination with the gentleMACS C-Tubes (Miltenyi Biotech, Auburn, CA). The cellularity included in the UGT samples is distributed in the epithelial layer, lamina propria, and stroma. The predominant immune cell composition of this compartment includes $\mathrm{T}$ cells, macrophages/dendritic cells, natural killer (NK) cells, neutrophils, and mast cells. Chlamydia infects primarily epithelial cells of the genital tract and we previously showed that the inflammation/fibrosis associated with Chlamydiainduced UGT pathologies is preceded by pathogenic EMT, which converts normal epithelial cells into fibroblastic mesenchymal cells $(8,30)$. Homogenized tissue samples were treated with QIAzol Lysis reagent (Qiagen, Germantown, $\mathrm{MD})$ and frozen at $-80^{\circ} \mathrm{C}$ until RNA extraction. Total RNA was isolated using the Qiagen miRNeasy Mini Kit (Qiagen, Germantown, MD) according to the manufacturer's instructions. Residual genomic DNA was removed by DNase I treatment (ThermoFisher Scientific). RNA integrity was assessed using the Agilent Technologies- 2100 Bioanalyzer and the concentration of purified RNA was determined using the NanoDrop ND-2000 Spectrophotometer (Thermo Fisher Scientific Inc., Wilmington, DE) according to the manufacturer's instructions. RNA samples were determined to have both an A260/280 and A260/230 ratio > 1.8. All samples were analyzed in triplicates.

\section{MiRNA Expression Profiling}

Purified RNA samples were analyzed using the NanoString nCounter Mouse miRNA Assay (NanoString Technologies, Seattle, WA). The assay involves a multiplexed hybridization reaction composed of up to 800 Reporter Probe sets (genes) and the RNA sample. Briefly, $100 \mathrm{ng}$ of each RNA sample was added to the miRNA-tag ligation reaction. Following 
ligation, samples were diluted 1:10, and 5ul each miRNAtag sample (equivalent to $10 \mathrm{ng}$ total RNA) were added to hybridizations and subjected to $3 \mathrm{~h}$ of automated processing per cartridge. Data acquisition was performed on a GEN2 Digital Analyzer and processed using NanoString's data analysis application nSolver. The nCounter miRNA v3 Assays included a set of six Positive and Negative control probes to monitor hybridization efficiency, Prep Station purification, and imaging. The Positive Control Performance data was expressed as the Linearity of Counts vs. RNA Concentration (Supplementary Figure 1). A set of Ligation miRNA controls were added to monitor miRNA-tag ligation efficiency. Endogenous miRNA counts were used to evaluate sample quality and data normalization.

\section{MicroRNA Data Analysis-Normalization, Principal Components Analysis, Hierarchical Clustering}

Each hybridization assay was normalized using the NanoString nSolver Analysis application based on the top-100 expressed miRNAs in each sample, filtered for those with raw counts $>$ 20. Normalization factors ranged between 0.1 and 10 for all assays. The positive top-100 miRNA normalized counts from each group were retained for further analysis. Target identification of genes putatively regulated by miRNAs was performed using TargetScan (Diana Tools-mirPath v.3) (31). Heatmaps were generated for differentially expressed miRNAs in each immunization group using R. Overlapping expression among miRNAs identified following immunization with the different vaccines was visualized using Venny 2.0, an interactive Venn diagram tool for comparing name lists of up to four genomic datasets (32). Subsequent Pathway, Core and Comparison analyses for all the false discovery rate (FDR)corrected differentially expressed miRNAs in infected and immunized mice were analyzed using the Ingenuity Pathway Analysis (IPA) software (Qiagen Inc.) and Diana MirPath (33). The top networks, molecular and cellular functions, diseases and disorders and physiological system development and functions were determined using Ingenuity System Interactive Pathway Systems (version 18,488,943). Stringency was set at "highly predicted" and "experimentally validated." The software uses its own internal algorithm and other databases, including TarBase, TargetScan, and microT-CDS, as well as findings published in the literature. Complete reference set for this analysis was carried out using the Ingenuity Knowledge Base (Genes + Endogenous Chemicals) for all molecules associated with diseases, functions, pathways, or list annotations.

\section{qRT-PCR}

Total RNA was isolated as indicated above and cDNA was synthesized using the miScript II RT Kit (Qiagen, Germantown, MD). qRT- PCR was performed on BioRad CFX96 system (BioRad, Hercules, CA) using specific miRNA primers and miScript SYBR Green PCR Kit (Qiagen) according to the manufacturer's protocol. The relative fold changes in miRNA expression were calculated by the delta-delta-cycle threshold $(\Delta \Delta \mathrm{Ct})$ method comparing miRNA expression levels in the genital tracts after immunization with the different vaccines. U6 miRNA was used as an endogenous control for expression normalization, and $\Delta \Delta \mathrm{Ct}$ was calculated as the difference between immunized and nonimmunized $\Delta \mathrm{Ct}$. The results are expressed as fold change, (the mean $\pm S D$ of three independent qRT-PCR runs), corresponding to $2^{-\Delta \Delta \mathrm{Ct}}$.

\section{Statistical Analysis}

The statistical analysis was performed using GraphPad Prism 8 software for Mac (GraphPad Software, Inc., La Jolla, CA, USA). Quantitative data were expressed as the mean \pm standard deviation $(S D)$, and the error bars indicate the SD from the mean. The statistical significance of the quantitative differences between any two groups was determined by the Student's twotailed $t$-test, and between three groups was assessed by analysis of variance (ANOVA). Statistical significance was determined at $p$-values $<0.05$.

\section{RESULTS}

\section{Profile of MiRNAs Expressed in the Upper Genital Tracts of Mice After Immunization and Infection}

MicroRNAs (mRNAs) with altered expression in the upper genital tracts of vaccine-immunized and live Chlamydia-infected mice were determined using a Nanostring quantitative assay platform. A total of 602 miRNAs were differentially expressed (DE) in the genital tracts of immunized and infected mice as compared to non-infected tissues. Of these, 58 miRNAs with counts $>100$ and $p$-values $<0.05$ were selected for further analysis. The proportion of miRNAs detected showed 41 (71\%) were detected in DC vaccine immunized mice, 23 (40\%) in VCG vaccine immunized mice, and 15 (26\%) in Chlamydia infected mice. Comparison of the 58 selected miRNAs using a Venn Diagram showed that only two miRNAs were common among the three groups, three between the DC vaccine and live Chlamydia infection groups and four between the VCG vaccine and Chlamydia infection groups (Figure 1A). Interestingly, we found that $10 \mathrm{DE}$ miRNAs were common between the DC and VCG vaccine groups (Figure 1A). The hierarchical clustering of the 58 differentially regulated miRNAs is shown in Figure 1B. Clustering was based on significance levels, with lighter colors representing higher significance values. In the DC vaccine group, miR-196b-5p and miR-3471 clustered together while miR-181a-5p and miR-10b-5p clustered together. In the VCG vaccine group, miR-539-5p and miR-2135 clustered together and were the most significantly upregulated miRNAs, while miR-350-3p and miR-222-3p clustered together in the live EB infected group. The clustering together of these miRNAs suggests they may exhibit similar pathway targeting patterns. The results further indicate that the diversity of up- or downregulated miRNA was different for each immunization and infection group (Tables 1-3). Thus, for the DC vaccine group, 
A

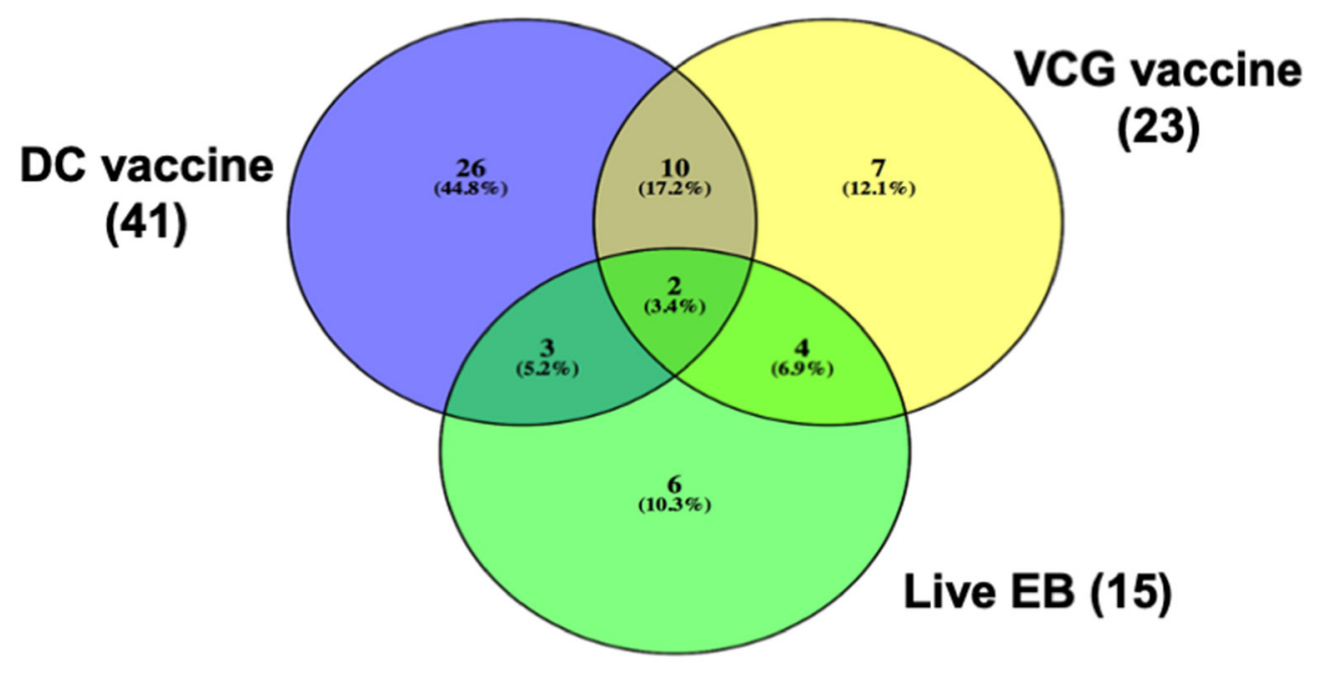

B

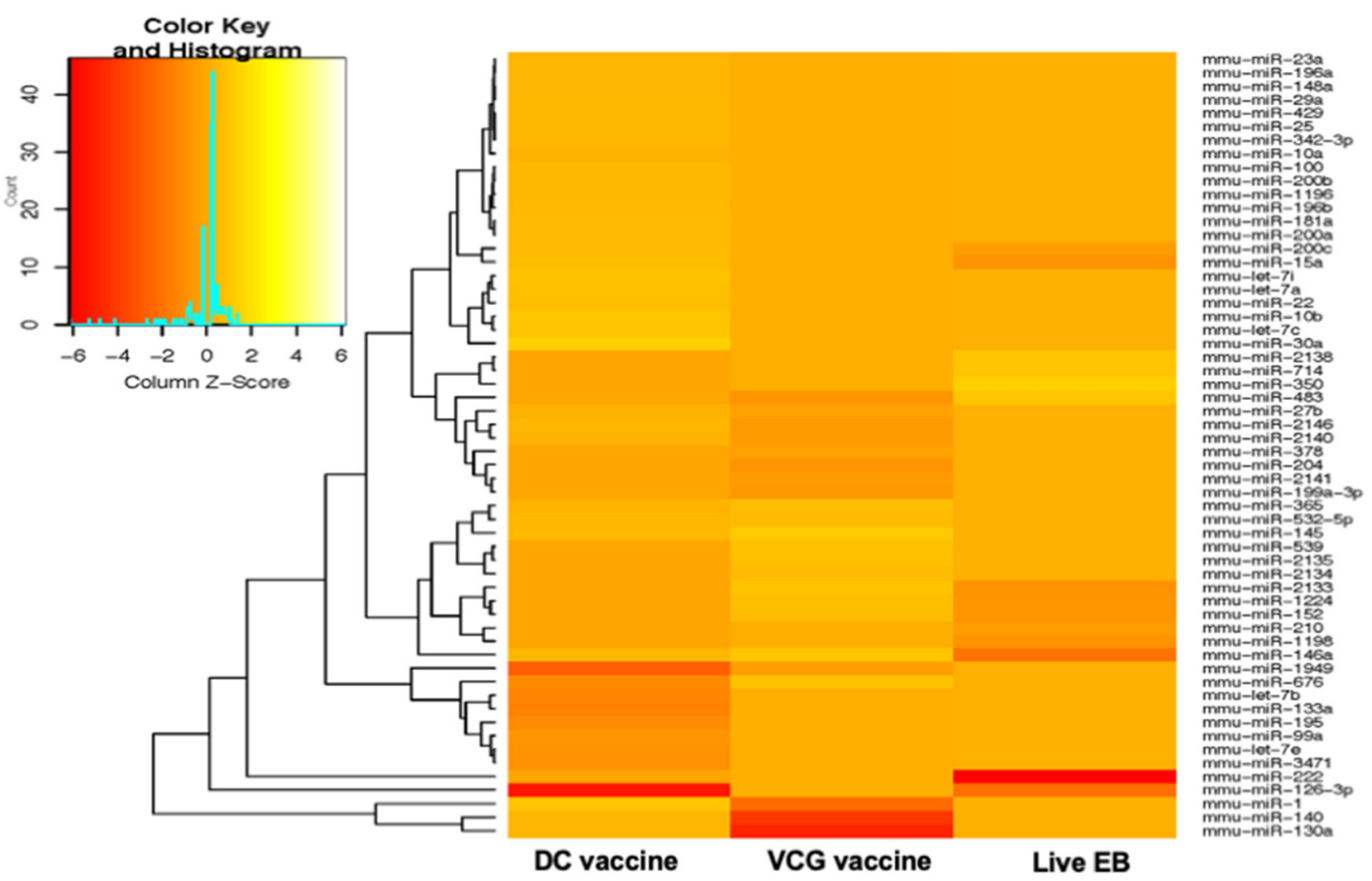

FIGURE 1 | Cluster and Heat map analysis of miRNAs significantly differentially expressed in UGT of mice after vaccine immunization and live Chlamydia infection. (A) Venn diagram of the top 58 upregulated and downregulated differentially expressed miRNAs after DC vaccine (41) or VCG vaccine (23) immunization and live Chlamydia infection (15). The numbers indicate the unique and common miRNAs in the immunization and infection groups. (B) Hierarchical cluster analysis of the top 58 upregulated and downregulated differentially expressed miRNAs after vaccine immunization and live Chlamydia infection. Each row represents a miRNA and each column, an immunization or infection group. On the scale of fold regulation, yellow corresponds to upregulated miRNAs, red corresponds to downregulated miRNAs, and brown indicates no expression difference.

32 miRNAs were upregulated, while nine were downregulated. Twelve miRNAs were upregulated and 11 were downregulated in the VCG vaccine group. In contrast with the DC and VCG vaccine groups, only four miRNAs were up-regulated and 11 were down-regulated in the live EB infection group. These results indicate that vaccine immunization and Chlamydia infection induces the DE of distinct miRNA profiles in the UGT of mice.

\section{Pattern of Up- and Down-Regulated MiRNAs Uniquely DE in the Upper Genital Tracts of Immunized and Infected Mice}

Twenty-one of the 32 up-regulated (including five members of the Let-7 family) and five of the nine down-regulated miRNAs were unique to the DC vaccinated mice (Figure 2A). On the other hand, only two of the 12 up-regulated and 
TABLE 1 | miRNAs DE expressed between DC-based vaccine and control.

\begin{tabular}{|c|c|c|c|}
\hline miRNA & Expression & Fold change & $P$-value* \\
\hline \multicolumn{4}{|l|}{ Upregulated (32) } \\
\hline mmu-miR-1a-3p & & 2.17 & 0.0196 \\
\hline *mmu-let-7a-5p & & 1.22 & 0.0344 \\
\hline${ }^{*} m m u-l e t-7 b-5 p$ & & 1.38 & 0.0085 \\
\hline *mmu-let-7c-5p & & 1.48 & 0.0039 \\
\hline *mmu-let-7e-5p & & 1.45 & 0.0190 \\
\hline *mmu-let-7i-5p & & 1.62 & 0.0155 \\
\hline *mmu-miR-10a-5p & & 1.45 & 0.0279 \\
\hline *mmu-miR-10b-5p & & 2.35 & 0.0003 \\
\hline *mmu-miR-22-3p & & 1.70 & 0.0129 \\
\hline *mmu-miR-23a-3p & & 1.18 & 0.0024 \\
\hline *mmu-miR-25-3p & & 1.37 & 0.0229 \\
\hline mmu-miR-27b-3p & & 2.76 & 0.0013 \\
\hline${ }^{*} m m u-m i R-29 a-3 p$ & & 1.33 & 0.0057 \\
\hline${ }^{*} m m u-m i R-30 a-5 p$ & & 1.26 & 0.0168 \\
\hline${ }^{*} m m u-m i R-99 a-5 p$ & & 1.65 & 0.0017 \\
\hline${ }^{*} m m u-m i R-100-5 p$ & & 1.58 & 0.0031 \\
\hline mmu-miR-126-3p & & 1.41 & 0.0129 \\
\hline mmu-miR-130a-3p & & 1.44 & 0.0394 \\
\hline *mmu-miR-133a-3p & & 1.47 & 0.0207 \\
\hline mmu-miR-140-5p & & 1.41 & 0.0203 \\
\hline mmu-miR-145-5p & & 1.85 & 0.0164 \\
\hline mmu-miR-146a-5p & & 1.87 & 0.0116 \\
\hline *mmu-miR-181a-5p & & 2.52 & 0.0012 \\
\hline${ }^{*} m m u-m i R-195 a-5 p$ & & 1.11 & 0.0224 \\
\hline *mmu-miR-196a-5p & & 2.04 & 0.0093 \\
\hline *mmu-miR-196b-5p & & 2.84 & 0.0029 \\
\hline *mmu-miR-342-3p & & 1.47 & 0.0379 \\
\hline mmu-miR-532-5p & & 1.47 & 0.0080 \\
\hline mmu-miR-365-3p & & 1.83 & 0.0155 \\
\hline mmu-miR-676-3p & & 1.74 & 0.0057 \\
\hline mmu-miR-1949 & & 2.72 & 0.0009 \\
\hline *mmu-miR-3471 & & 3.67 & 0.0256 \\
\hline \multicolumn{4}{|l|}{ Downregulated (9) } \\
\hline mmu-miR-15a-5p & & -1.43 & 0.0500 \\
\hline${ }^{*} m m u-m i R-148 a-3 p$ & & -1.63 & 0.0010 \\
\hline${ }^{*} m m u-m i R-200 a-3 p$ & & -3.11 & 0.0056 \\
\hline *mmu-miR-200b-3p & & -2.22 & 0.0123 \\
\hline mmu-miR-200c-3p & Anti-inflammatory & -2.37 & 0.0467 \\
\hline *mmu-miR-429-3p & & -2.85 & 0.0007 \\
\hline *mmu-miR-1196 & & -1.66 & 0.0322 \\
\hline mmu-miR-2140 & & -6.02 & 0.0118 \\
\hline mmu-miR-2146 & & -12.21 & 0.0034 \\
\hline
\end{tabular}

*Expressed in DC vaccine only.

five of the 11 down-regulated miRNAs were unique to the VCG vaccinated mice (Figure 2B). Among these, miR-2135 (upregulated) and miR-2134 (down-regulated) have not previously been reported in the literature. In addition, two of the four up-regulated and four of the 11 down-regulated miRNAs were unique to the live EB group (Figure 2C). Many of these
TABLE 2 | miRNAs DE expressed between VCG-based vaccine and control.

\begin{tabular}{|c|c|c|c|}
\hline miRNA & Expression & Fold change & $P$-value* \\
\hline \multicolumn{4}{|l|}{ Upregulated (12) } \\
\hline mmu-miR-1a-3p & & 2.17 & 0.0196 \\
\hline mmu-miR-1a-3p & & 1.73 & 0.0486 \\
\hline mmu-miR-27b-3p & & 2.30 & 0.0032 \\
\hline mmu-miR-130a-3p & & 1.22 & 0.0113 \\
\hline mmu-miR-140-5p & & 1.28 & 0.0476 \\
\hline mmu-miR-145a-5p & & 1.53 & 0.0414 \\
\hline mmu-miR-146a-5p & & 1.53 & 0.0189 \\
\hline mmu-miR-532-5p & & 1.31 & 0.0051 \\
\hline${ }^{*} m m u-m i R-539-5 p$ & & 1.88 & 0.0105 \\
\hline mmu-miR-365-3p & & 1.81 & 0.0484 \\
\hline mmu-miR-676-3p & & 1.56 & 0.0014 \\
\hline mmu-miR-1949 & & 2.54 & 0.0031 \\
\hline${ }^{*} m m u-m i R-2135$ & & 1.66 & 0.0128 \\
\hline \multicolumn{4}{|l|}{ Downregulated (11) } \\
\hline mmu-miR-152-3p & & -1.47 & 0.0053 \\
\hline${ }^{*} m m u-m i R-199 a-3 p$ & & -1.31 & 0.0419 \\
\hline${ }^{*} m m u-m i R-204-5 p$ & & -2.24 & 0.0060 \\
\hline${ }^{*} m m u-m i R-378 a-3 p$ & & -1.24 & 0.0242 \\
\hline mmu-miR-483-5p & & -2.04 & 0.0187 \\
\hline mmu-miR-1224-5p & & -2.49 & 0.0427 \\
\hline mmu-miR-2133 & & -13.56 & 0.0018 \\
\hline${ }^{*} m m u-m i R-2134$ & & -2.53 & 0.0067 \\
\hline mmu-miR-2140 & & -6.79 & 0.0002 \\
\hline${ }^{*} m m u-m i R-2141$ & & -2.22 & 0.0241 \\
\hline mmu-miR-2146 & & -11.69 & 0.0021 \\
\hline
\end{tabular}

${ }^{*}$ Expressed in VCG vaccine only.

miRNAs with $\log$ fold changes ranging from-13.56 to 3.67 have been shown to have human homologs. Comparative profiling also showed that nine of the upregulated (let-7a$5 p$, let-7c-5p, let-7e-5p, miR-23a-3p, miR-25a-3p, miR-29a-3p, miR-181a-5p, miR-195a-5p, and miR-196a-5p) and 4 of the downregulated (miR-148a-3p, miR-200a-3p, miR-200b-3p, and miR-429-3p) miRs uniquely DE in the UGT of DC vaccineimmunized mice were inflammation and immunopathologyassociated (Table 4) $(8,30,35)$. The results revealed that many of the uniquely DE miRNAs in the UGT of DC vaccine-immunized mice were associated with inflammation and immunopathology.

\section{Pattern of Up- and Down-Regulated DE MiRNAs Common Between Infected and the Different Immunization Groups}

Only one inflammation and immunopathology-associated miRNA (miR-146a) was commonly expressed among the immunized and infected groups (Figure 3A). In addition, four inflammation and immunopathology-associated miRs (miR-27b, miR-130a, miR-140, and miR-145) were commonly expressed in the genital tracts of DC and VCG vaccine immunized mice (Figure 3B). Of the two miRNAs commonly expressed in the 
TABLE 3 | miRNAs DE expressed between Chlamydia infection and control.

\begin{tabular}{|c|c|c|c|}
\hline miRNA & Expression & Fold change & $P$-value* \\
\hline \multicolumn{4}{|l|}{ Upregulated (4) } \\
\hline mmu-miR-126a-3p & & 1.31 & 0.0456 \\
\hline mmu-miR-146a-5p & & 1.42 & 0.0347 \\
\hline *mmu-miR-222-3p & & 1.52 & 0.0452 \\
\hline *mmu-miR-350-3p & & 2.17 & 0.0437 \\
\hline \multicolumn{4}{|l|}{ Downregulated (11) } \\
\hline mmu-miR-15a-5p & & -1.84 & 0.0036 \\
\hline mmu-miR-152-3p & & -1.31 & 0.0261 \\
\hline mmu-miR-200c-3p & & -2.00 & 0.0239 \\
\hline *mmu-miR-210-3p & & -1.98 & 0.0033 \\
\hline mmu-miR-483-5p & & -1.86 & 0.0191 \\
\hline *mmu-miR-714 & & -2.02 & 0.0305 \\
\hline *mmu-miR-1198-5p & & -1.34 & 0.0287 \\
\hline mmu-miR-1224-5p & & -4.03 & 0.0118 \\
\hline mmu-miR-2133 & & -12.16 & 0.0404 \\
\hline *mmu-miR-2138 & & -2.96 & 0.0366 \\
\hline mmu-miR-2140 & & -4.43 & 0.0075 \\
\hline
\end{tabular}

${ }^{*}$ Expressed in live Chlamydia infection only.

genital tracts of infected and immunization groups, miR-146a was up-regulated while miR-2140, which has only previously been reported in the salivary glands of ticks after a blood meal (50), was down-regulated (Figure 3A). Among the 10 miRNAs (mmu-miR-1a-3p, mmu-miR-130a-3p, mmu-miR140-5p, mmu-miR-145-5p, mmu-miR-1949, mmu-miR-2146, mmu-miR-27b-3p mmu-miR-365-3p, mmu-miR-532-5p, and mmu-miR-676-3p) commonly expressed in DC and VCG vaccine immunized mice, only mmu-miR-2146 was downregulated while the rest were up-regulated (Figure 3B). Of the three miRNAs commonly expressed in the genital tracts of DC vaccine immunized and Chlamydia infected mice, miR-126-3p was up-regulated while miR-15a and miR-200c were down-regulated (Figure 3C). All four miRNAs (miR-1224, miR-152, miR-2133, and miR-483) commonly expressed in the VCG vaccine-immunized and Chlamydia infected mice were down-regulated (Figure 3D). Our findings reveal that five of the DE miRNAs (miR-2134, miR-2135, miR-2138, miR-2140, and miR-2146) are novel; these have not previously been characterized in the context of infection or vaccination.

\section{qPCR Data Validation}

To validate the differential expression of miRNAs identified by the Nanostring quantitative assay platform, we selected four DE miRNAs (miR-146, 126, 15a, and 200c) for qPCR analysis. The miR-146 was selected from the two miRNAs that were commonly expressed in all groups of mice after immunization and infection. The remaining three miRNAs were those that were common between the DC vaccine and Chlamydia infected groups. The results showed that the data generated by the Nanostring assay and qPCR were consistent in terms of up- or down-regulation and differed only in the magnitude of the response measured by the two approaches (Figure 4 and Supplementary Figure 2).

\section{MiRNAs DE in the Upper Genital Tracts of Mice After Infection and Immunization Regulate a Number of Pathways Involved in Disease and Biological Functions}

To evaluate the biological pathways activated in the upper genital tracts of mice following infection and immunization, we performed core analysis of the DE miRNAs in the framework of biological processes and molecular networks (diseases and disorders, molecular and cellular functions, and physiological system development) using IPA. In pathways associated with diseases and disorders, there were substantial differences in the number of differentially expressed miRNAs regulating immunological and inflammatory diseases, inflammatory response, endocrine system disorders, infectious diseases, cancer and developmental disorder after Chlamydia infection and immunization with DC and VCG vaccines (Figure 4A). In the case of molecular and cellular functions-associated pathways, there were differences in the number of differentially expressed miRNAs regulating cell cycle, cellular development, cellular growth and proliferation, cellular movement, cellular assembly and organization, cellular function and maintenance, and cellular compromise between the infection and immunization groups (Figure 4B). Also, there were substantial differences in the number of differentially expressed miRNAs regulating embryonic development, organismal development, connective tissue development and function, and lymphoid tissue structure pathways associated with physiological system development and function after Chlamydia infection and immunization with DC and VCG vaccines (Figure 4C).

We generated KEGG pathways of the differentially expressed miRNAs unique to each infection and immunization group using the Diana Tools-mirPath v.3 (33). Based on the DIANATarBase reference database, a higher number of biological pathways were predicted to be targeted by miRNAs differentially expressed in the UGT of mice after immunization with the DC vaccine compared to the VCG vaccine and Chlamydia infection groups. For the DC vaccine group, 27 pathways were predicted to be regulated by the DE miRNAs (Figure 5A). The pathways included MAPK, ErbB, HIF-1B, mTOR, Pathways in cancer, Glioma, FoxO, Chronic myeloid leukemia, Renal cell carcinoma, Hepatitis B, Colorectal cancer, Protein processing in the endoplasmic reticulum, Fatty acid metabolism, ECMreceptor interaction, and Fatty acid biosynthesis. For the VCG vaccine group, six pathways were predicted to be regulated by the differentially expressed miRNAs (Figure 5B), including signaling pathways regulating pluripotency of stem cells, Hippo signaling, TGF-beta signaling, Estrogen signaling, lysine degradation, and gap junction. For the Chlamydia infection group, three pathways were predicted to be regulated by the differentially expressed miRNAs (Figure 5C) and included lysine degradation, Hippo signaling and ECM-receptor interaction. Of these biological pathways, the mTOR, FoxO, MAPK, HIF-1, ErbB, Hippo, TGF-beta, ECM-receptor interaction, and Estrogen signaling pathways were identified as important markers of immunity and inflammation. We selected specific KEGG pathways regulated by miRNAs DE in the upper genital tract in response to DC vaccine 

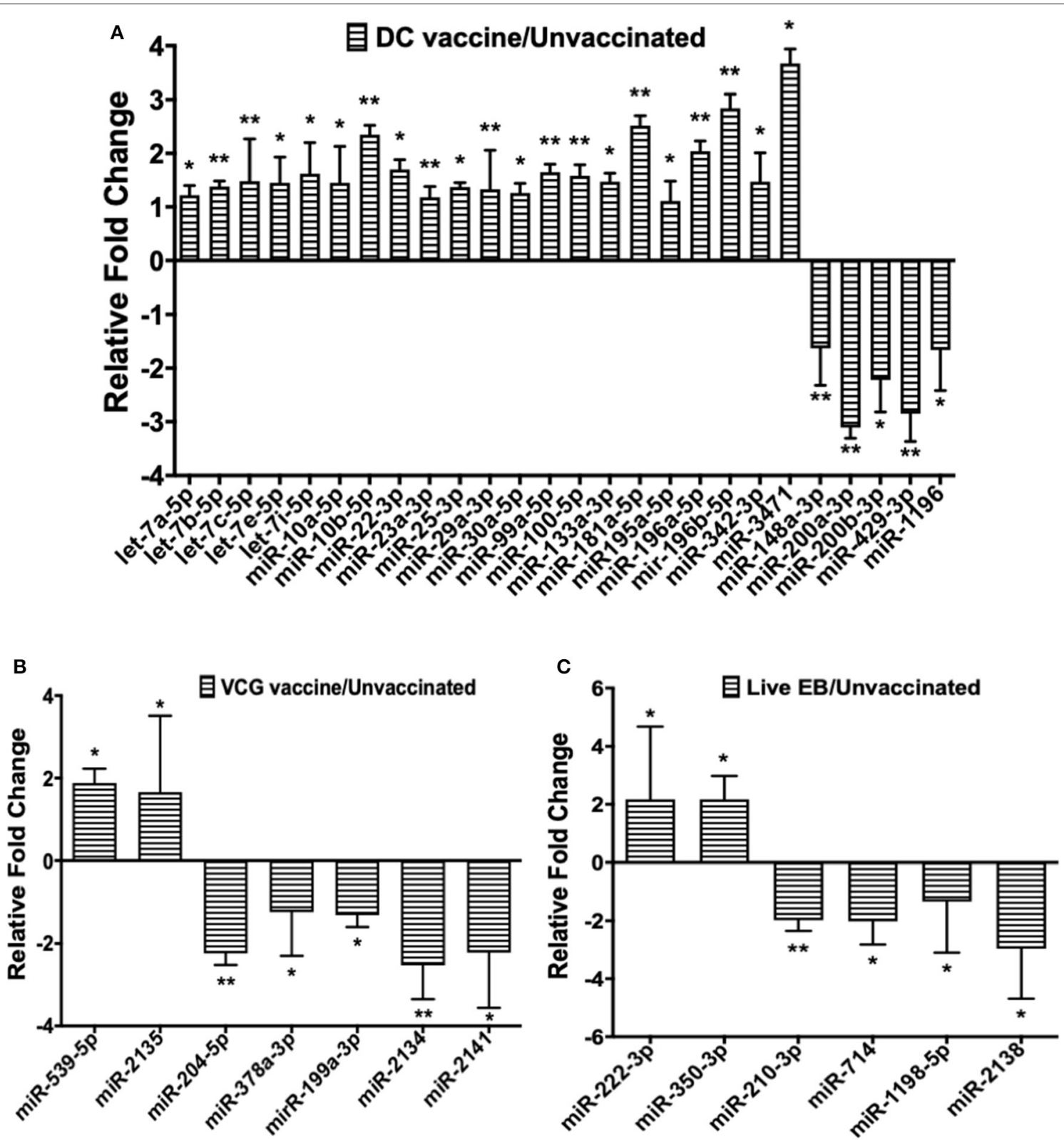

FIGURE 2 | Quantification of miRNAs uniquely differentially expressed in the UGT of mice after (A) DC vaccine immunization, (B) VCG vaccine immunization, and (C) live Chlamydia infection. miRNAs were normalized using the NanoString nSolver Analysis application based on the top-100 expressed miRNAs in each sample, filtered for those with raw counts $>20$. Each reaction was performed with triplicate samples, and the data is shown as the mean for each group. Results are expressed as relative fold changes in miRNA expression compared to non-immunized controls. Statistical analysis was performed by the NanoString nSolver Analysis software and significant levels were simultaneously generated with the data. ${ }^{*} p<0.05,{ }^{* *} p<0.01$.

immunization to buttress the significance of these pathways in immune response, host cell modification, and cell to cell communication (Supplementary Figures 3A-G).

Further evaluation of the top networks associated with differentially expressed miRNAs using IPA showed that for the DC vaccine, two networks were generated; Network 1 consists of DE miRNAs and 15 focus molecules associated with Cancer, Organismal Injury and Abnormalities, and Cell Cycle. E2F Transcription Factor 1 (E2F1), a transcription regulator, was the central focus molecule associated with the miRNAs (Figure 6A). Network 2 consists of miRNAs and molecules associated with Energy Production, Lipid Metabolism, and Small Molecule Biochemistry. There were seven focus molecules, with the transcription regulator, Peroxisome ProliferatorActivated Receptor Alpha (PPARA) being the central focus molecule associated with miRNAs (Figure 6B). The VCG vaccine generated one associated network consisting of DE miRNAs and molecules associated with Cellular Development, Cellular 
TABLE 4 | Inflammation and immunity-associated miRNAs uniquely differentially expressed (DE) in the UGT of DC vaccine-immunized mice.

\begin{tabular}{|c|c|c|c|c|c|c|}
\hline DE miRNA & $\begin{array}{l}\text { Fold- } \\
\text { change }\end{array}$ & Targets & $P$-value & $\begin{array}{l}\text { Biological } \\
\text { function/disease }\end{array}$ & $\begin{array}{l}\text { Anti/pro- } \\
\text { inflammatory }\end{array}$ & References \\
\hline let-7a-5p & 1.22 & IL-6, HMGA1/2 & 0.0344 & $\begin{array}{l}\text { Downregulating } \\
\text { Inflammation }\end{array}$ & Anti-Inflammatory & (34) \\
\hline Let-7c-5p & 1.48 & Caspase 3, N-RAS, MMP1 & 0.0039 & $\begin{array}{l}\text { Downregulating } \\
\text { Inflammation }\end{array}$ & Anti-Inflammatory & $(35,36)$ \\
\hline Let-7e-5p & 1.45 & TLR4 & 0.0190 & $\begin{array}{l}\text { Downregulating } \\
\text { Inflammation }\end{array}$ & Anti-Inflammatory & $(37)$ \\
\hline miR-23a-3p & 1.18 & $\begin{array}{l}1 \mathrm{KK} \alpha \\
\mathrm{CiGadd} 45 \mathrm{ab}\end{array}$ & 0.0024 & $\begin{array}{l}\text { Innate immune responses } \\
\text { Inflammation/Apoptosis }\end{array}$ & $\begin{array}{l}\text { Anti-Inflammatory } \\
\text { Pro-Inflammatory }\end{array}$ & $(37,38)$ \\
\hline miR-25-3p & 1.37 & Adam10 & 0.0229 & $\begin{array}{l}\text { Downregulating } \\
\text { inflammation }\end{array}$ & Anti-Inflammatory & (39) \\
\hline miR-29a-3p & 1.33 & $\begin{array}{l}\text { TET1, MUC1 } \\
\text { TRIM68, CDC4 } \\
\text { MMP2 }\end{array}$ & 0.0057 & $\begin{array}{l}\text { Inflammation/tumor } \\
\text { suppressor } \\
\text { Cell migration/proliferation } \\
\text { Apoptosis }\end{array}$ & $\begin{array}{l}\text { Anti-Inflammatory } \\
- \\
-\end{array}$ & $(40)$ \\
\hline miR-181a-5p & 2.52 & $\begin{array}{l}\text { MMP-14 } \\
\text { Ago2, Smad2/3 }\end{array}$ & 0.0012 & $\begin{array}{l}\text { Innate immune responses } \\
\text { Cell migration/proliferation } \\
\text { EMT/tumor suppressor }\end{array}$ & $\begin{array}{l}\text { Anti-Inflammatory } \\
\text { Anti-Inflammatory } \\
\text { Pro-inflammatory }\end{array}$ & $(30,41,42)$ \\
\hline miR-195a-5p & 1.11 & $\begin{array}{l}\text { Smad7, TRIM14 } \\
\text { Cyclin E1 }\end{array}$ & 0.0224 & $\begin{array}{l}\text { Cell proliferation/migration } \\
\text { Tumor suppressor }\end{array}$ & $\begin{array}{l}- \\
-\end{array}$ & $(43)$ \\
\hline miR-196a-5p & 2.04 & $\begin{array}{l}\text { MAP4K3, IKK } \alpha \\
\text { ELOVL1 }\end{array}$ & 0.0093 & $\begin{array}{l}\text { Downregulating } \\
\text { inflammation } \\
\text { Migration/EMT }\end{array}$ & $\begin{array}{l}\text { Anti-Inflammatory } \\
-\end{array}$ & $(44)$ \\
\hline miR-148a-3p & -1.63 & PTEN/AKT & 0.0010 & $\begin{array}{l}\text { Downregulating } \\
\text { inflammation }\end{array}$ & Anti-Inflammatory & $(45)$ \\
\hline miR-200a-3p & -3.11 & E-cadherin, QKI, ZEB1/2 & 0.0056 & $\begin{array}{l}\text { EMT induction } \\
\text { Cell proliferation/migration }\end{array}$ & $\begin{array}{l}\text { Pro-Inflammatory } \\
\text { - }\end{array}$ & $(46,47)$ \\
\hline miR-200b-3p & -2.22 & E-cadherin, QKI, ZEB1/2 & 0.0123 & EMT induction & Pro-Inflammatory & $(8,48)$ \\
\hline miR-429-3p & -2.85 & $\begin{array}{l}\text { ZEB1 } \\
\text { CRKL }\end{array}$ & 0.0007 & $\begin{array}{l}\text { EMT induction } \\
\text { Cell proliferation/migration }\end{array}$ & $\begin{array}{l}\text { Pro-Inflammatory } \\
\text { - }\end{array}$ & $(8,49)$ \\
\hline
\end{tabular}

Growth and Proliferation, and Organ Development with nine focus molecules. Interferon-gamma (IFNG), a cytokine/growth factor, was the central molecule associated with miRNAs (Figure 6C). The Chlamydia infection group generated one associated network consisting of Cell Cycle, Connective Tissue Development and Function, and Embryonic Development. There were 34 focus molecules and the transcription regulator, E2F1, was the central molecule associated with miRNAs (Figure 6D). Together, these results demonstrate that miRNAs DE in the upper genital tracts of mice after infection and immunization regulate pathways involved in disease and biological functions.

\section{DISCUSSION}

We investigated if the differential miRNA profiles induced in the UGT of mice correlate with the disparate immunity vs. pathologic outcomes associated with vaccine immunization and chlamydial infection. The study shows that immunization with select chlamydial vaccines or infection with live Chlamydia drives differing miRNA levels and diversity in the UGT of mice. Among the miRNAs uniquely expressed in each of the experimental groups, we observed that miRNAs elicited by the DC vaccine were more likely to be upregulated than down regulated. In contrast, miRNAs in the infection group were more likely to be down regulated. The finding of significant differences in the number and diversity of up- and down-regulated miRNAs uniquely DE in the upper genital tracts of vaccine immunized and Chlamydia infected mice indicates that the miRNA profile expressed by immunization differs substantially from that expressed following infection. Differential expression of miRNAs during Chlamydia genital infection has previously been associated with immune response and pathogenesis $(8,16$, 18, 24, 26).

Interestingly, the two miRNAs common to all the three treatment groups were all differentially expressed in the same direction, indicating that these miRNAs may be significant in some of the changes associated with protection against pathology. One of these miRNAs, miR-146a, is a crucial regulator of the innate immune response whose overexpression suppresses hepatic stellate cell proliferation and activation and inhibits LPS-induced pro-inflammatory cytokine secretion (51). Also, miR-146a attenuates liver fibrosis by directly suppressing the profibrogenic effects of transforming growth factor- $\beta 1$ (TGF $\beta 1$ ) (52). Our finding of the expression of miR-2134, miR-2135, miR2138 , miR-2140, and miR-2146 following infection or vaccination is novel. The only report of miR-2140 has been in the salivary glands of ticks after a blood meal (50). In general, all the differentially expressed miRNAs common to the vaccine groups 

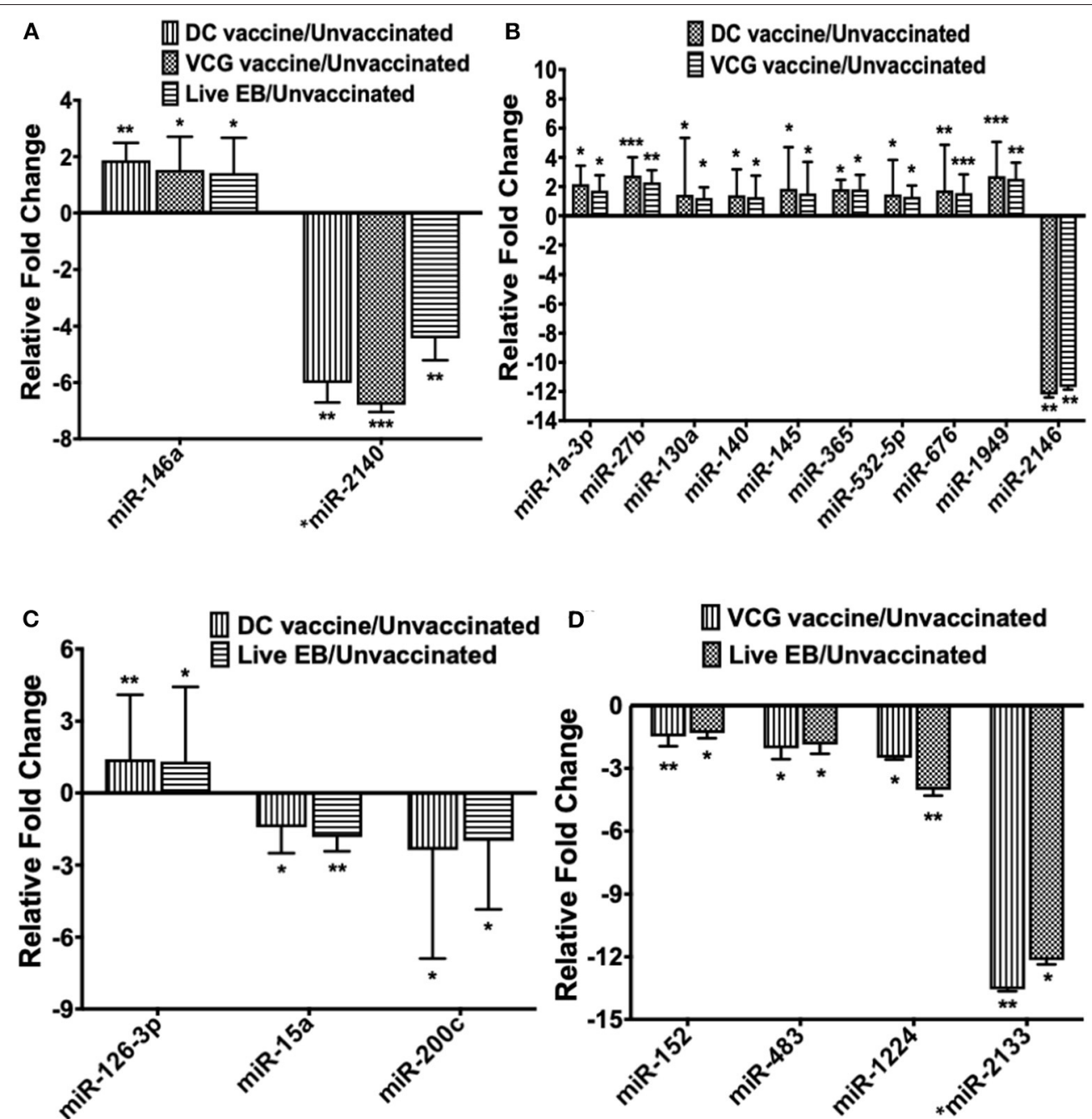

FIGURE 3 | The pattern of DE miRNAs in UGT of mice common between infected and immunized mice. (A) Levels of differentially expressed miRNA common between the immunization (DC and VCG vaccines) and live Chlamydia infection groups. (B) Levels of differentially expressed miRNA common between DC and VCG vaccine groups. (C) Levels of differentially expressed miRNA common between DC vaccine-immunized and live Chlamydia infection groups. (D) Levels of differentially expressed miRNA common between VCG vaccine-immunized and live Chlamydia infection groups. Data, which are the means from three animals per group, are plotted as fold change relative to non-immunized control levels. Significant differences between three groups were evaluated by one-way analysis of variance (ANOVA) with Tukey's post multiple comparison test and between two groups by Student's $t$-test at ${ }^{\star} p<0.05,{ }^{\star \star} p<0.01,{ }^{\star \star \star} p<0.001$.

were in the same direction, either upregulated or downregulated, implying that they are likely to regulate the same pathways. It also appears differences between the vaccines are more in the nature or unique miRNAs found in each vaccine group than in similar groups. Using four select miRNAs, we also validated the miRNA expression changes identified by the Nanostring quantitative assay platform, demonstrating a strong correlation between NanoString and the qPCR methods.

We observed the differential expression of many inflammation and immunopathology-associated miRNAs following immunization and infection. The role of these miRNAs in bacterial infectious diseases involves the modulation of inflammatory responses, tissue remodeling, and innate and adaptive immunity (53). Increasing evidence shows that inflammatory-related miRNAs play essential roles in maintaining fertility formation, eliminating cancer, and development of the male reproductive tract (54). One of the inflammation-associated miRNAs, miR-146a, is induced by different pro-inflammatory stimuli, such as IL-1 $\beta$ and TNF- $\alpha$ (55, 56), and is upregulated in various human pathologies associated with activation of inflammatory responses $(57,58)$. Also, the expression of miR-146a reportedly increased after Helicobacter pylori infection in an NF-кB-dependent manner (53). This increase may inhibit the expression of IL-8, macrophage inflammatory protein (MIP)-3 $\alpha$, TNF- $\alpha$, and IL1 $\beta$ by reducing NF- $\kappa$ B activity $(59,60)$. These strongly support the role of miR-146 in the regulation of inflammation and may represent a potential 


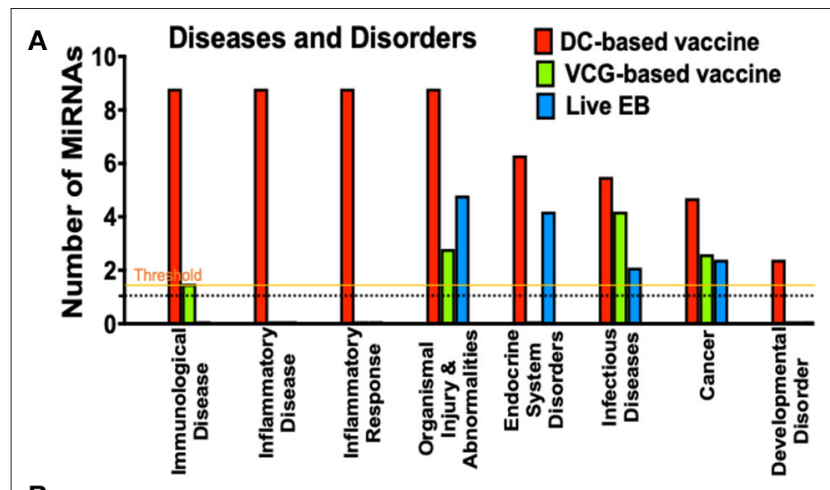

B

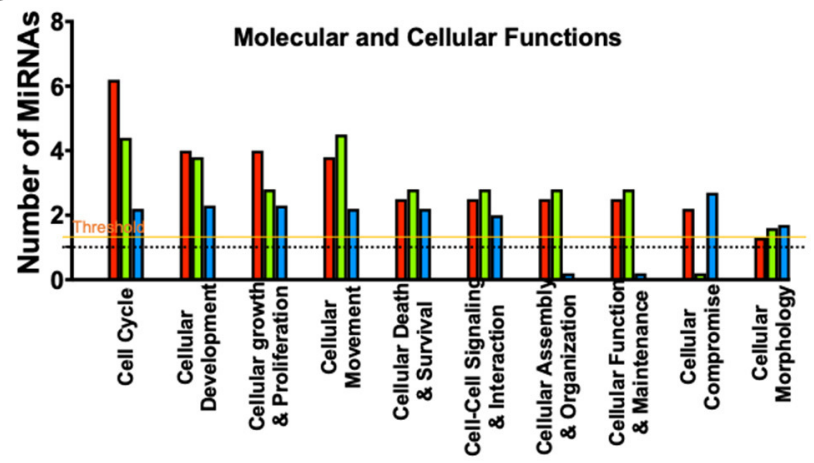

C

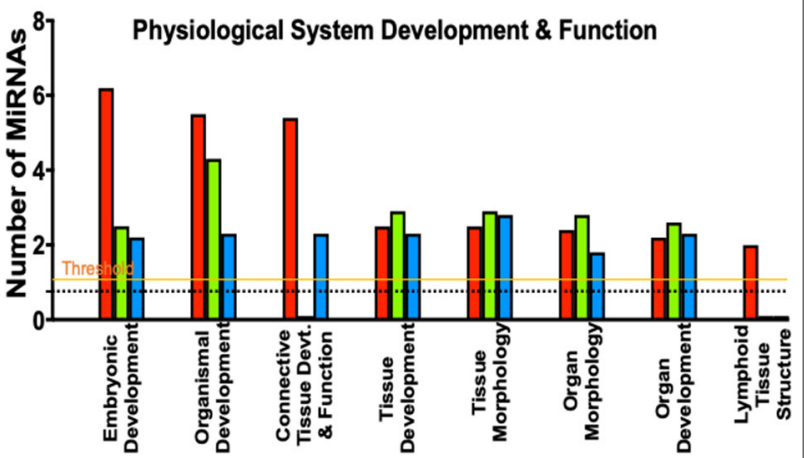

FIGURE 4 | A comparison of canonical pathways predicted to be regulated by miRNAs differentially expressed in the UGT of mice after immunization and live Chlamydia infection. (A) Pathways predicted to regulate diseases and disorders after vaccine immunization and Chlamydia infection. (B) Pathways predicted to regulate molecular and cellular functions after vaccine immunization and Chlamydia infection. (C) Pathways predicted to regulate physiological system development and function after vaccine immunization and Chlamydia infection.

tool for therapeutic intervention in inflammatory mediated pathological disease conditions. Other inflammation and immunopathology-associated miRNAs uniquely $\mathrm{DE}$ in the UGT of DC vaccine-immunized mice, including miR-23a$3 p$, miR-25a-3p, and miR-29a-3p have been reported to be involved in the induction of innate immune responses, inflammation, cell migration/proliferation, apoptosis, and tumor suppression (37-40).

Of the biological pathways predicted by KEGG pathway analysis to be targeted by miRNAs differentially expressed after immunization and infection, mTOR, FoxO, MAPK, HIF-1, ErbB, Hippo, TGF-beta, ECM-receptor interaction, and Estrogen signaling pathways are significant markers of immunity and inflammation. We previously showed that downregulation of certain miRNAs, such as miR-15a and miR27a targeted pathways, including the TGF-beta pathway to prevent fibrogenesis (30). Here, the TGF-beta pathway was predicted to be regulated by miR-204-5p, which was uniquely DE (downregulated) following VCG vaccine immunization. It is therefore conceivable that miR-204-5p controls fibrosis and maintains epithelial integrity in the UGT by specifically targeting TGF-beta signaling. Thus, although TGF-beta is known to play a crucial role in the pathogenesis of fibrosis, it also plays a significant role in tissue homeostasis, immunity and cell proliferation (61). Previous studies reported that the Forkhead transcription factor 1 (FoxO1) regulates genes involved in apoptosis and autophagy, anti-oxidative enzymes, cell cycle arrest genes, and metabolic and immune regulators $(62,63)$. The FoxO1/3 proteins are well-established anti-proliferation and proapoptotic factors that have favorable inhibitory effects on fibroblast activation and subsequent extracellular matrix (ECM) production capable of ameliorating fibrosis in organs such as the heart, liver, lungs, and kidneys (64). Therefore, it has been suggested that inhibition of fibroblast activation may be a promising anti-fibrosis therapeutic strategy (65). Fibrosis is the excessive formation of fibrous connective tissue (66) resulting from copious amounts of extracellular matrix (ECM) molecules and is essential for wound healing and maintenance of structural integrity $(67,68)$. FoxO and ECM production, known to prevent Chlamydia-induced fibrosis and infertility, are two biological pathways predicted in our study to be targeted by DC vaccineinduced miRNAs $(8,9)$. An additional interesting finding from this study is the observation that the mammalian target of rapamycin (mTOR) pathway was only targeted by DC vaccineinduced miRNAs. The beneficial role of mTOR in modulating inflammation and autophagy under liver ischemia/reperfusion (IR) injury has recently been reviewed (69). mTOR is an evolutionarily conserved serine/threonine protein kinase that plays a vital role in regulating mRNA translation, metabolism, and protein turnover (70). An excessive inflammatory response is a crucial mechanism of liver IR injury, and overexpression of mTOR in the liver significantly reduces liver inflammation and apoptosis (71). Furthermore, mTOR-deficient mice express higher levels of inflammation-related genes such as MCP1 , TNF- $\alpha$, and IL- 6 than wild-type mice after liver IR by negatively modulating NF- $\mathrm{KB}$ (71). These results suggest that the FoxO, mTOR, and ECM pathways or miRNAs targeting them might be vital targets for the therapy and prevention of Chlamydia-induced fibrosis, which subsequently leads to infertility and could guide the development of therapeutic agents for ameliorating Chlamydia-induced fibrosis in the female upper genital tract.

Several genes were directly or indirectly regulated by several miRNAs, some of which are associated with inflammation and immunopathology. For example, Ago2 and Smad2/3 were predicted targets of miR-181a-5p, and loss of Smad3 blocks EMT, leading to fibrotic sequelae (30). miR-181a, a member 
A DC vaccine
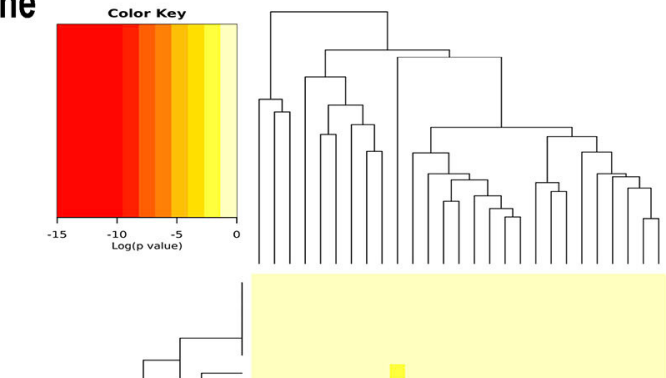

nmu-mik-1966-5p|Tarbase mmu-miR-347171Tarbase mmu-mir-100-5p|Trarbase Mmu-miR-1952-5p TTarbase mmu-miR-429-3p|Tarbase mmu-miR-342-3p Trarbase mmu-miR-148a-3p Tharbase mmu-milR-99a-5p|Tarbase mmu-miR-2002-3p|Trarbase mmulete.70-5p|Tarbase mmu-let-7a-5p|Tarbase mmu-mik-23a-3p|larbase
mmu-mik-30a-50|Tarbase mmu-mik-30-5olTarbase mmu-let.7c.5p prarbase mmu-let-7i-5p|Tarbase mmu-miR-22-3p|Trarbase mmu-mili-29a-30|Tarbase mmu-mik-10b-5p|Tarbase mmu-mik-25-3p|tarbase

B VCG vaccine

c Live EB
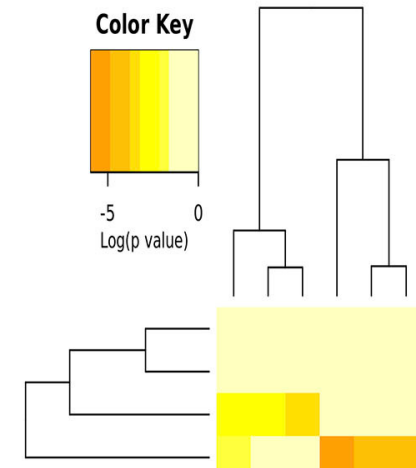

Color Key
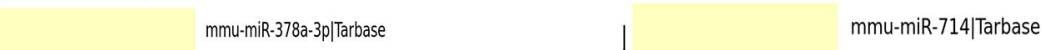

mmu-miR-539-5p|Tarbase
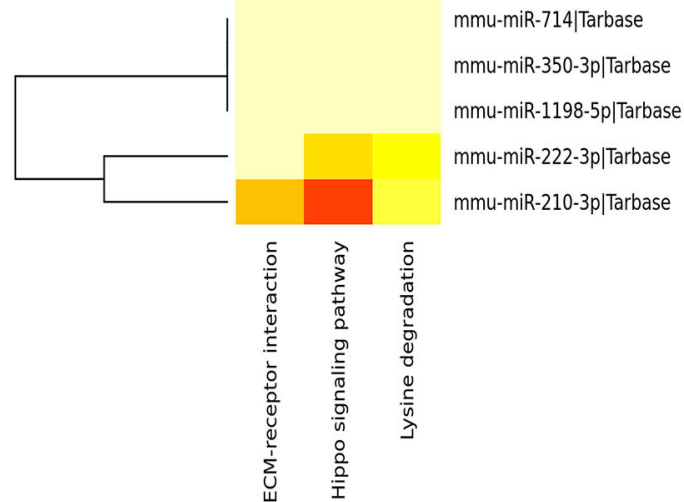

FIGURE 5 | Significance cluster analysis showing pathways predicted to be regulated by miRNAs differentially expressed in the UGT of mice after immunization with DC vaccine (A), VCG vaccine (B), and live Chlamydia infection (C). The hierarchical clustering results for miRNAs are shown on the right Y-axis and pathways on the X-axis. On the miRNA axis, we can identify miRNAs clustered together by exhibiting similar pathway targeting patterns. Similar clustering can also be seen on the pathway axis. Darker colors represent lower significance values.

of the miR-181 family, regulates immune function and plays a significant role in innate and adaptive immune responses (41). In cancer studies, miR-181a-5p inhibits cancer cell migration and angiogenesis via downregulation of matrix metalloproteinase14 (MMP-14) (42). More recently, miR-181a was reported to be involved in the modulation of $\mathrm{CD}^{+} \mathrm{T}$ cell activation and 

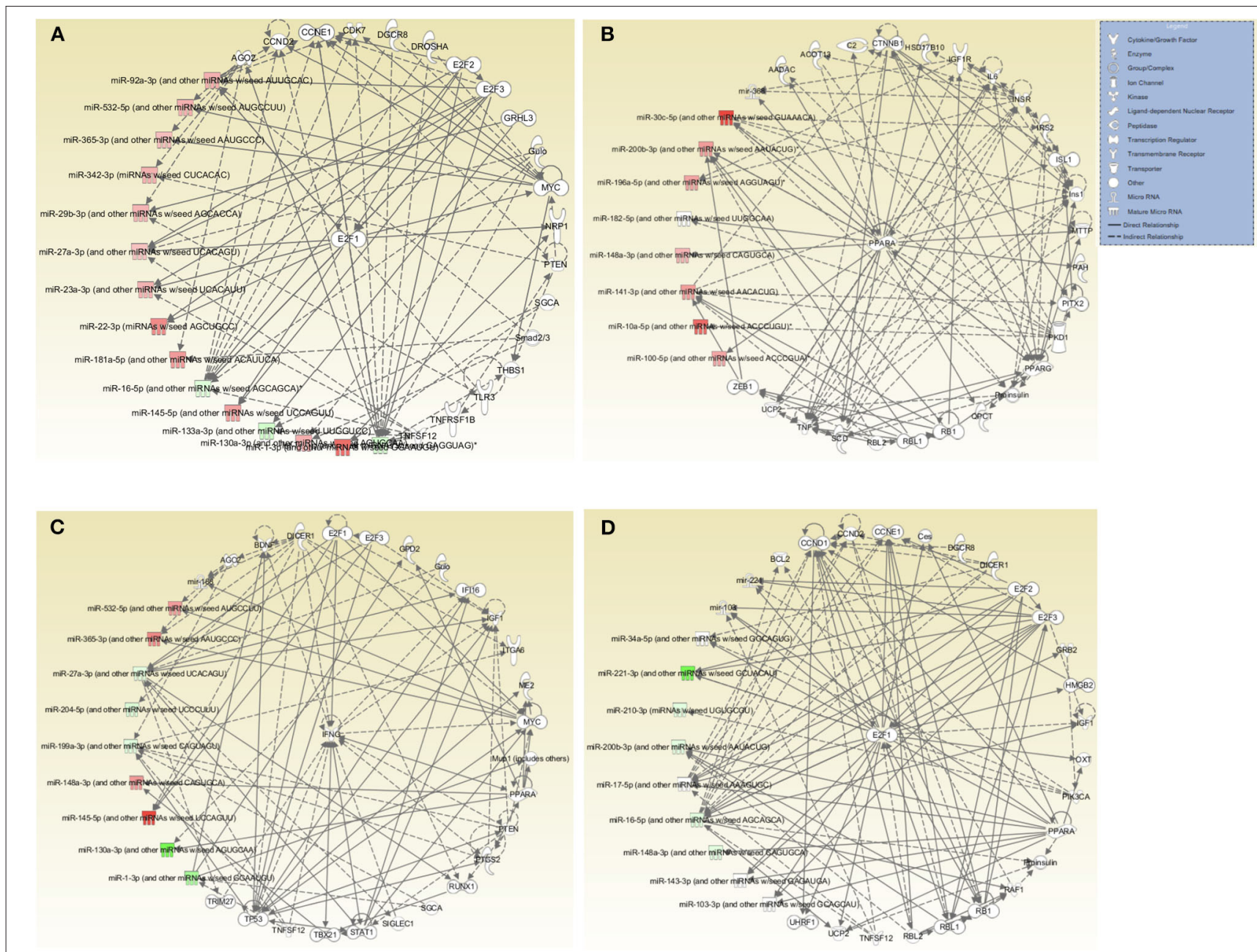

FIGURE 6 | Networks associated with differentially expressed miRNAs. (A) Network One - Cancer, Organismal Injury and Abnormalities, and Cell Cycle associated with miRNAs DE in the genital tract of mice immunized with DC-based Vaccine. E2F1, a transcription regulator, is the central molecule associated with miRNAs. (B) Network Two - Energy Production, Lipid Metabolism, Small Molecule Biochemistry associated with miRNAs DE in the genital tract of mice immunized with the DC-based Vaccine. The transcription regulator, PPARA, is the central molecule associated with miRNAs. (C) Cellular Development, Cellular Growth and Proliferation and Organ Development Network associated with miRNAs DE in the genital tract of mice immunized with the VCG-based Chlamydia Vaccine. IFNG, a cytokine/growth factor, is the central molecule associated with miRNAs. (D) Cell Cycle, Embryonic Development, Connective Tissue Development and Function Network associated with miRNAs DE in the genital tract of mice infected with Live Chlamydia. The transcription regulator E2F1 is the central molecule associated with miRNAs.

plasticity and decreased the expression level of the Th1-related transcription factor T-bet and the Th17-related transcription factor ROR $\gamma$ t (72). Also, mir-204-5p, which was downregulated following immunization with VCG vaccine was associated with Dicer 1, a ribonuclease III enzyme whose loss in mouse oviducts results in significant reproductive abnormalities (15). Chlamydia infection alters the expression of crucial miRNAs that regulate the integrity of Dicer, EMT, fibrosis, and tumorigenesis in the reproductive system $(8,15)$. We recently showed that the upregulation of mir-204-5p following Chlamydia infection leads to genital tract pathology (23). Another miRNA, miR$146 \mathrm{a}$, in addition to being directly regulated by the E2F2 and E2F3 transcription factors, was also indirectly regulated by Dicer 1. miR-146a, a member of the inflammation-inducible miRNA family, negatively regulates Toll-like receptor (TLR) signaling (73) and has been reported to modulate innate immune responses during bacterial infection by targeting IRAK1 and TRAF6 (55). It also negatively regulated NF- $\mathrm{KB}$ activation by inhibiting IRAK1 and TRAF6 expression, significantly inhibiting breast tumor growth (74). The dysregulation of these miRNAs following vaccine immunization strongly suggests their involvement in protection against UGT pathology by regulating Dicer 1 expression. ZEB1, RBL1, RB1, and PPARA were direct targets of miR-200b-3p. Previous studies have described the role of miR-200 family members, including miR-200a, miR200b, miR-200c, and miR-429-3p in the regulation of expression of the E-cadherin transcriptional repressors ZEB1, ZEB2, and CRKL previously implicated in EMT and tumor metastasis $(8,46-49)$. We previously showed that the downregulation of miR-200a, miR-200b, miR-200c, and miR-429-3p following 
genital Chlamydia infection resulted in EMT induction by targeting ZEB1 (8). Our study confirms the downregulation of miR-200c after Chlamydia infection and further shows that immunization with the DC vaccine also downregulates miR-200a, miR-200b, miR-200c, and miR-429-3p. Thus, the miR-200 family members act as negative regulators of ZEB1, RBL1, RB1, and PPARA expression leading to processes that contribute to the disruption of the epithelial functional integrity of the UGT and induction of EMT and fibrosis. Thus, EMT, in addition to tumor invasion and metastasis, also plays a crucial role in embryonic development, wound healing, and tissue fibrosis. We found that Ago2 and CCNE1 were direct targets of members of the Let-7 family that were upregulated following immunization with the DC vaccine. Several members of the Let-7 family, including let-7a-5p, let-7c-5p, and let$7 e-5 p$ have previously been reported to inhibit migration, invasion and EMT by targeting IL-6, HMGA1/2, Caspase 3, N-RAS, MMP1, and TLR4 (34-37). Although our study did not identify any direct targets for miR-195a-5p and IGF1R and INSR were only indirectly associated with miR-196a-5p, previous studies have reported the involvement of these miRs with cell proliferation/migration, EMT and tumor suppression by targeting several genes, including Smad7, TRIM14, Cyclin E1, MAP4K3, and IKK $\alpha(43,44)$. In addition, we showed that PPARA was associated with the downregulation of mir-148a$3 p$ whose overexpression has been reported to enhance M1 macrophage polarization leading to pro-inflammatory responses and enhanced ability to kill bacteria by targeting PTEN/AKT (45). Although expression levels of target genes or molecules of miRNAs differentially expressed in the UGT of vaccinated and infected mice was not evaluated in this study, we previously reported the altered expression of proteins that regulate epithelial functional integrity, EMT and tumorigenesis in the UGT following Chlamydia infection (8). Several of these proteins were identified to be targets of miRNAs that control epithelial integrity through EMT. Taken together, the results indicate that the up- or down-regulation of these miRs plays a significant role in the induction of innate and adaptive immune responses leading to protective immunity or immunopathology by regulating genes directly or indirectly associated with immunity and immunopathology.

In conclusion, we have demonstrated that vaccine immunization and Chlamydia infection induced the expression of distinct miRNA profiles in the mouse UGT and targeted genes that regulate several biological processes and functions associated with immune response and inflammation. The results strongly suggest that these unique miRNA profiles play a significant role in the disparate immunity outcomes associated with Chlamydia infection and vaccination through regulation

\section{REFERENCES}

1. Westrom L, Joesoef R, Reynolds G, Hagdu A, Thompson S. Pelvic inflammatory disease and fertility. A cohort study of 1,844 women with laparoscopically verified disease and 657 control women with of their target genes in combination with diverse biological processes and pathways. Further studies will investigate the molecular mechanisms linking these miRs with their gene targets and how they modulate immunity and immunopathology following Chlamydia infection and vaccination.

\section{DATA AVAILABILITY STATEMENT}

The original contributions presented in the study are included in the article/Supplementary Material, further inquiries can be directed to the corresponding author/s.

\section{ETHICS STATEMENT}

The animal study was reviewed and approved by The Institutional Animal Care and Use Committee (IACUC) of Morehouse School of Medicine (MSM) (Assurance number A3381-01).

\section{AUTHOR CONTRIBUTIONS}

FE and $\mathrm{YO}$ conceived and designed the study. SH and SR performed most of the experiments. KD, FM, and SL contributed to the experimental work. IB, FE, and YO made substantial contributions to analysis and interpretation of data. $\mathrm{OA}$ generated Figure 1B. JI and YO contributed to data interpretation and critically revised the manuscript. FE wrote the manuscript. All authors have read and agreed to the publication of the manuscript.

\section{FUNDING}

This research was funded by the National Institutes of Health (R01AI126897) to FE. The investigation was conducted in a facility constructed with support from Research Facilities Improvement Grant \#1 C06 RR18386 from the National Center for Research Resources, National Institutes of Health.

\section{ACKNOWLEDGMENTS}

The authors sincerely thank Babayewa Oguljahan for her research support. The technical support of the Core facility staff is also gratefully acknowledged.

\section{SUPPLEMENTARY MATERIAL}

The Supplementary Material for this article can be found online at: https://www.frontiersin.org/articles/10.3389/fimmu. 2021.625318/full\#supplementary-material 
3. De La Maza L, Zhong G, Brunham R. Update on Chlamydia trachomatis vaccinology. Clin Vaccine Immunol. (2017) 24:e00543-16. doi: 10.1128/CVI.00543-16

4. Soper D. Pelvic inflammatory disease. Obstet Gynecol. (2010) 116:419-28. doi: 10.1097/AOG.0b013e3181e92c54

5. Workowski K, Bolan G. Sexually transmitted diseases treatment guidelines. MMWR Recomm Rep. (2015) 64:1-137.

6. Park S, Lee S, Kim M, Kang Y, Moon H, Rhim C. Clinical characteristics of genital chlamydia infection in pelvic inflammatory disease BMC Womens Health. (2017) 17 5. doi: 10.1186/s12905-016-0356-9

7. Hafner LM. Pathogenesis of fallopian tube damage caused by Chlamydia trachomatis infections. Contraception. (2015) 92:108-15. doi: 10.1016/j.contraception.2015.01.004

8. Igietseme J, Omosun Y, Stuchlik O, Reed M, Partin J, He Q, et al. Role of epithelial-mesenchyme transition in chlamydia pathogenesis. PLOS ONE. (2015) 10 e0145198. doi: 10.1371/journal.pone.0145198

9. Igietseme JU, He Q, Joseph K, Eko FO, Lyn D, Ananaba G, et al. Role of T lymphocytes in the pathogenesis of Chlamydia disease. J Infect Dis. (2009) 200:926-34. doi: 10.1086/605411

10. Pais R, Omosun Y, He Q, Blas-Machado U, Black C, Igietseme JU, et al. Rectal administration of a chlamydial subunit vaccine protects against genital infection and upper reproductive tract pathology in mice. PLOS ONE. (2017) 12:e178537. doi: 10.1371/journal.pone.0178537

11. Igietseme JU, Ananaba GA, Bolier J, Bowers S, Moore T, Belay T, et al. Suppression of endogenous IL-10 gene expression in dendritic cells enhances antigen presentation for enhanced specific Th1 induction: potential for cellular vaccine development. J Immunol. (2000) 164:4212-9. doi: 10.4049/jimmunol.164.8.4212

12. Muller T, Becker E, Stallmann S, Waldhuber A, Rommler-Dreher F, Albrecht $\mathrm{S}$, et al. Vaccination with the polymorphic membrane protein A reduces Chlamydia muridarum induced genital tract pathology. Vaccine. (2017) 35:2801-10. doi: 10.1016/j.vaccine.2017.04.017

13. Starnbach MN, Roan NR. Conquering sexually transmitted diseases. Nat Rev Immunol. (2008) 8:313-7. doi: 10.1038/nri2272

14. Vicetti Miguel RD, Quispe Calla NE, Pavelko SD, Cherpes TL. Intravaginal Chlamydia trachomatis challenge infection elicits TH1 and TH17 immune responses in mice that promote pathogen clearance and genital tract damage. PLoS ONE. (2016) 11:e0162445. doi: 10.1371/journal.pone.0162445

15. Igietseme JU, Omosun Y, Partin J, Goldstein J, He Q, Joseph K, et al. Prevention of Chlamydia-induced infertility by inhibition of local caspase activity. J Infect Dis. (2013) 207:1095-104. doi: 10.1093/infdis/jit009

16. Gupta R, Arkatkar T, Yu, JJ, Wali S, Haskins W, Chambers J, et al. Chlamydia muridarum infection associated host microRNAs in the murine genital tract and contribution to generation of host immune response. Am J Reprod Immunol. (2015) 73:126-40. doi: 10.1111/aji.12281

17. Stallmann S, Hegemann J. The Chlamydia trachomatis Ctad1 invasin exploits the human integrin $\beta 1$ receptor for host cell entry. Cell Microbiol. (2016) 18:761-75. doi: 10.1111/cmi.12549

18. Yeruva L, Pouncey D, Eledge M, Bhattacharya S, Luo C, Weatherford E, et al. MicroRNAs modulate pathogenesis resulting from chlamydial infection in mice. Infect Immun. (2016) 85:e00768-16. doi: 10.1128/IAI.00768-16

19. Bartel DP. MicroRNAs: genomics, biogenesis, mechanism, and function. Cell. (2004) 116:281-97. doi: 10.1016/S0092-8674(04)00045-5

20. Skalsky RL, Cullen BR. Viruses, microRNAs, and host interactions. Annu Rev Microbiol. (2010) 64:123-41. doi: 10.1146/annurev.micro.112408.134243

21. Krek A, Grun D, Poy MN, Wolf R, Rosenberg L, Epstein EJ, et al. Combinatorial microRNA target predictions. Nat Genet. (2005) 37:495-500. doi: $10.1038 /$ ng 1536

22. Shyu A, Wilkinson M, Van Hoof A. Messenger RNA regulation: to translate or to degrade. EMBO J. (2008) 27:471-81. doi: 10.1038/sj.emboj.7601977

23. Benyeogor I, Simoneaux T, Wu Y, Lundy S, George Z, Ryans K, et al. A unique insight into the MiRNA profile during genital chlamydial infection. BMC Genom. (2019) 20:143. doi: 10.1186/s12864-019-5495-6

24. Arkatkar T, Gupta R, Li W, Yu J-J, Wali S, Guentzel M, et al. Murine MicroRNA-214 regulates intracellular adhesion molecule (ICAM1) gene expression in genital Chlamydia muridarum infection. Immunology. (2015) 145:534-42. doi: 10.1111/imm.12470
25. Derrick T, Last A, Burr S, Roberts C, Nabicassa M, Cassama E, et al. Inverse relationship between microRNA-155 and-184 expression with increasing conjunctival inflammation during ocular Chlamydia trachomatis infection. BMC Infect Dis. (2016) 16:8. doi: 10.1186/s12879-016-1367-8

26. Gupta R, Arkatkar T, Keck J, Koundinya GK, Castillo K, Hobel S, et al. Antigen specific immune response in Chlamydia muridarum genital infection is dependent on murine microRNAs-155 and-182. Oncotarget. (2016) 7:6472642. doi: 10.18632/oncotarget.11461

27. Yeruva L, Myers G, Spencer N, Creasy H, Adams N, Maurelli A, et al. Early microRNA expression profile as a prognostic biomarker for the development of pelvic inflammatory disease in a mouse model of chlamydial genital infection. mBio. (2014) 5:e01241-14. doi: 10.1128/mBio.01241-14

28. Eko FO, Mayr UB, Attridge SR, Lubitz W. Characterization and immunogenicity of Vibrio cholerae ghosts expressing toxin-coregulated pili. JBiotechnol. (2000) 83:115-23. doi: 10.1016/S0168-1656(00)00315-1

29. Eko F, Lubitz W, Mcmillan L, Ramey K, Moore T, Ananaba GA, et al. Recombinant Vibrio cholerae ghosts as a delivery vehicle for vaccinating against Chlamydia trachomatis. Vaccine. (2003) 21:1694-703. doi: 10.1016/S0264-410X(02)00677-1

30. Igietseme JU, Omosun Y, Nagy T, Stuchlik O, Reed MS, He Q, et al. Molecular pathogenesis of chlamydia disease complications: epithelial-mesenchymal transition and fibrosis. Infect Immun. (2018) 86:17. doi: 10.1128/IAI.00585-17

31. Agarwal V, Bell GW, Nam JW, Bartel DP. Predicting effective microRNA target sites in mammalian mRNAs. Elife. (2015) 4:28. doi: 10.7554/eLife.05005.028

32. Oliveros J. Venny. An interactive tool for comparing lists with Venn's diagrams. (2007-2015).

33. Vlachos IS, Zagganas K, Paraskevopoulou MD, Georgakilas G, Karagkouni D, Vergoulis T, et al. (2015). DIANA-miRPath v3.0: deciphering microRNA function with experimental support. Nucl Acids Res. 43, W460-6. doi: 10.1093/nar/gkv403

34. Wu A, Wu K, Li J, Mo Y, Lin Y, Wang Y, et al. Let-7a inhibits migration, invasion and epithelial-mesenchymal transition by targeting HMGA2 in nasopharyngeal carcinoma. J Transl Med. (2015) 13:105. doi: 10.1186/s12967-015-0462-8

35. Johnson SM, Grosshans H, Shingara J, Byrom M, Jarvis R, Cheng A, et al. RAS is regulated by the let-7 microRNA family. Cell. (2005) 120:635-47. doi: 10.1016/j.cell.2005.01.014

36. Lv J, Zeng Y, Qian Y, Dong J, Zhang Z, Zhang J. MicroRNA let-7c-5p improves neurological outcomes in a murine model of traumatic brain injury by suppressing neuroinflammation and regulating microglial activation. Brain Res. (2018) 1685:1691-104. doi: 10.1016/j.brainres.2018.01.032

37. Nejad C, Stunden HJ, Gantier MP. A guide to miRNAs in inflammation and innate immune responses. FEBS J. (2018) 285:3695-716. doi: $10.1111 /$ febs. 14482

38. Fang Y, Xu XY, Shen Y, Li J. miR-23a-3p and miR-23a-5p target CiGadd45ab to modulate inflammatory response and apoptosis in grass carp. Fish Shellfish Immunol. (2020) 98:34-44. doi: 10.1016/j.fsi.2019.12.076

39. Yao Y, Sun W, Sun Q, Jing B, Liu S, Liu X, et al. Platelet-derived exosomal microRNA-25-3p inhibits coronary vascular endothelial cell inflammation through Adam10 via the NF-кB signaling pathway in ApoE(-/-) mice. Front Immunol. (2019) 10:2205. doi: 10.3389/fimmu.2019.02205

40. Wang J-Y, Zhang Q, Wang D-D, Yan W, Sha H-H, Zhao J-H, et al. MiR-29a: a potential therapeutic target and promising biomarker in tumors. Biosci Rep. (2018) 38:1265. doi: 10.1042/BSR20171265

41. Fu G, Rybakin V, Brzostek J, Paster W, Acuto O, Gascoigne NR. Fine-tuning T cell receptor signaling to control T cell development. Trends Immunol. (2014) 35:311-8. doi: 10.1016/j.it.2014.05.003

42. Li Y, Kuscu C, Banach A, Zhang Q, Pulkoski-Gross A, Kim D, et al. miR-181a-5p inhibits cancer cell migration and angiogenesis via downregulation of matrix metalloproteinase-14. Cancer Res. (2015) 75:267485. doi: 10.1158/0008-5472.CAN-14-2875

43. Yu W, Liang X, Li X, Zhang Y, Sun Z, Liu Y, et al. MicroRNA-195: a review of its role in cancers. Onco Targets Ther. (2018) 11:7109-23. doi: 10.2147/OTT.S183600

44. Shah N, Singh I. MicroRNA profiling identifies miR-196a as differentially expressed in childhood adrenoleukodystrophy and 
adult adrenomyeloneuropathy. Mol Neurobiol. (2017) 54:1392-403. doi: 10.1007/s12035-016-9746-0

45. Huang F, Zhao J-L, Wang L, Gao C-C, Liang S-Q, An D-J, et al. miR-148a-3p mediates notch signaling to promote the differentiation and M1 activation of macrophages. Front Immunol. (2017) 8:1327. doi: 10.3389/fimmu.2017.01327

46. Gregory PA, Bert AG, Paterson EL, Barry SC, Tsykin A, Farshid G, et al. The miR-200 family and miR-205 regulate epithelial to mesenchymal transition by targeting ZEB1 and SIP1. Nat Cell Biol. (2008) 10:593-601. doi: $10.1038 /$ ncb 1722

47. Feng X, Wang Z, Fillmore R, Xi Y. MiR-200, a new star miRNA in human cancer. Cancer Lett. (2014) 344:166-73. doi: 10.1016/j.canlet.2013.11.004

48. Kurata A, Yamada M, Ohno S, Inoue S, Hashimoto H, Fujita K, et al. Expression level of microRNA-200c is associated with cell morphology in vitro and histological differentiation through regulation of ZEB1/2 and E-cadherin in gastric carcinoma. Oncol Rep. (2018) 39:91-100. doi: 10.3892/or.2017.6093

49. Guo C, Zhao D, Zhang Q, Liu S, Sun M-Z. miR-429 suppresses tumor migration and invasion by targeting CRKL in hepatocellular carcinoma via inhibiting Raf/MEK/ERK pathway and epithelial-mesenchymal transition. Sci Rep. (2018) 8:2375. doi: 10.1038/s41598-018-20258-8

50. Zhou J, Zhou Y, Cao J, Zhang H, Yu Y. Distinctive microRNA profiles in the salivary glands of Haemaphysalis longicornis related to tick blood-feeding. Exp Appl Acarol. (2013) 59:339-49. doi: 10.1007/s10493-012-9604-3

51. Chen Y, Zeng Z, Shen X, Wu Z, Dong Y, Cheng JC. MicroRNA-146a-5p negatively regulates pro-inflammatory cytokine secretion and cell activation in lipopolysaccharide stimulated human hepatic stellate cells through inhibition of toll-like receptor 4 signaling pathways. Int J Mol Sci. (2016) 17:71076. doi: 10.3390/ijms17071076

52. Zou Y, Cai Y, Lu D, Zhou Y, Yao Q, Zhang S. MicroRNA-146a$5 p$ attenuates liver fibrosis by suppressing profibrogenic effects of TGFbetal and lipopolysaccharide. Cell Signal. (2017) 39:1-8. doi: 10.1016/j.cellsig.2017.07.016

53. Zhou $\mathrm{X}$, Li X, Wu M. miRNAs reshape immunity and inflammatory responses in bacterial infection. Signal Transduct Target Ther. (2018) 3:14. doi: 10.1038/s41392-018-0006-9

54. Parker MI, Palladino MA. MicroRNAs downregulated following immune activation of rat testis. Am J Reprod Immunol. (2017) 77:12673. doi: 10.1111/aji.12673

55. Taganov KD, Boldin MP, Chang KJ, Baltimore D. NF-kappaB-dependent induction of microRNA miR-146, an inhibitor targeted to signaling proteins of innate immune responses. Proc Natl Acad Sci USA. (2006) 103:12481-6. doi: 10.1073/pnas.0605298103

56. Sheedy FJ, O'neill LA. Adding fuel to fire: microRNAs as a new class of mediators of inflammation. Ann Rheum Dis. (2008) 67(Suppl.3):iii50-55. doi: $10.1136 /$ ard.2008.100289

57. Nakasa T, Miyaki S, Okubo A, Hashimoto M, Nishida K, Ochi M, et al. Expression of microRNA-146 in rheumatoid arthritis synovial tissue. Arthritis Rheum. (2008) 58:1284-92. doi: 10.1002/art.23429

58. Sonkoly E, Stahle M, Pivarcsi A. MicroRNAs and immunity: novel players in the regulation of normal immune function and inflammation. Semin Cancer Biol. (2008) 18:131-40. doi: 10.1016/j.semcancer.2008.01.005

59. Liu Z, Xiao B, Tang B, Li B, Li N, Zhu E, et al. Up-regulated microRNA146a negatively modulate Helicobacter pylori-induced inflammatory response in human gastric epithelial cells. Microbes Infect. (2010) 12:854-63. doi: 10.1016/j.micinf.2010.06.002

60. Li N, Xu X, Xiao B, Zhu ED, Li BS, Liu Z, et al. H. pylori related proinflammatory cytokines contribute to the induction of miR- 146a in human gastric epithelial cells. Mol Biol Rep. (2012) 39:4655-61. doi: 10.1007/s11033-011-1257-5

61. Biernacka A, Dobaczewski M, Frangogiannis NG. TGF- $\beta$ signaling in fibrosis. Growth Fact. (2011) 29:196-202. doi: 10.3109/08977194.2011.595714

62. Wang S, Xia P, Huang G, Zhu P, Liu J, Ye B, et al. FoxO1-mediated autophagy is required for NK cell development and innate immunity. Nat Commun. (2016) 11023:11023. doi: 10.1038/ncomms 11023

63. Murtaza G, Khan A, Rashid R, Muneer S, Hasan S, Chen J. FOXO transcriptional factors and long-term living. Oxid Med Cell Longev. (2017) 2017:3494289. doi: 10.1155/2017/3494289

64. Xin Z, Ma Z, Hu W, Jiang S, Yang Z, Li T, et al. FOXO1/3: potential suppressors of fibrosis. Ageing Res Rev. (2018) 41:42-52. doi: 10.1016/j.arr.2017.11.002

65. Guo J, Guan Q, Liu X, Wang H, Gleave ME, Nguan CY, et al. Relationship of clusterin with renal inflammation and fibrosis after the recovery phase of ischemia-reperfusion injury. BMC Nephrol. (2016) 17:133. doi: 10.1186/s12882-016-0348-x

66. O'reilly S. Epigenetics in fibrosis. Mol Aspects Med. (2017) 54:89-102. doi: 10.1016/j.mam.2016.10.001

67. Eming SA, Martin P, Tomic-Canic M. Wound repair and regeneration: mechanisms, signaling, and translation. Sci Transl Med. (2014) 6:265sr266. doi: 10.1126/scitranslmed.3009337

68. Lindsey M, Iyer R, Zamilpa R, Yabluchanskiy A, Deleon-Pennell K, Hall M, et al. A novel collagen matricryptin reduces left ventricular dilation postmyocardial infarction by promoting scar formation and angiogenesis. J Am College Cardiol. (2015) 66:1364-74. doi: 10.1016/j.jacc.2015.07.035

69. Zhang T, Guo J, Gu J, Chen K, Li H, Wang J. Protective role of mTOR in liver ischemia/reperfusion injury: involvement of inflammation and autophagy. Oxid Med Cell Longev. (2019) 2019:7861290. doi: 10.1155/2019/78 61290

70. Saxton RA, Sabatini DM. mTOR signaling in growth, metabolism, and disease. Cell. (2017) 168:960-76. doi: 10.1016/j.cell.2017.02.004

71. Li Z, Zhang J, Mulholland M, Zhang W. mTOR activation protects liver from ischemia/reperfusion-induced injury through NF-kappaB pathway. Faseb J. (2017) 31:3018-26. doi: 10.1096/fj.201601278R

72. Liu X, Luo M, Meng H, Zeng Q, Xu L, Hu B, et al. MiR-181a regulates CD4+ $\mathrm{T}$ cell activation and differentiation by targeting IL-2 in the pathogenesis of myasthenia gravis. Eur J Immunol. (2020). doi: 10.1002/eji.201848007

73. Ordas A, Kanwal Z, Lindenberg V, Rougeot J, Mink M, Spaink HP, et al. MicroRNA-146 function in the innate immune transcriptome response of zebrafish embryos to Salmonella typhimurium infection. BMC Genom. (2013) 14:696. doi: 10.1186/1471-2164-14-696

74. Liu R, Liu C, Chen D, Yang WH, Liu X, Liu CG, et al. FOXP3 controls an miR$146 /$ NF- $\kappa B$ negative feedback loop that inhibits apoptosis in breast cancer cells. Cancer Res. (2015) 75:1703-13. doi: 10.1158/0008-5472.CAN-14-2108

Conflict of Interest: The authors declare that the research was conducted in the absence of any commercial or financial relationships that could be construed as a potential conflict of interest.

Copyright (๑) 2021 Howard, Richardson, Benyeogor, Omosun, Dye, Medhavi, Lundy, Adebayo, Igietseme and Eko. This is an open-access article distributed under the terms of the Creative Commons Attribution License (CC BY). The use, distribution or reproduction in other forums is permitted, provided the original author(s) and the copyright owner(s) are credited and that the original publication in this journal is cited, in accordance with accepted academic practice. No use, distribution or reproduction is permitted which does not comply with these terms. 